\title{
1 A longitudinal examination of students' health behaviours during their first year at \\ 2 university
}

\section{Abstract}

4 Background: During the transition from school to higher education, young adults experience

5 a substantial amount of change where they progress from the highly controlled setting of

6 school to the autonomous and self-motivated environment of university. Time spent at

7 university is considered a critical period during which young adults establish a clear sense of

8 identity, which can include forming positive health behaviours such as regular physical

9 activity. The transition from school to university also involves a number of changes that can impact on the mental well-being of students including financial concerns, academic pressures and a lack of social support Aim: To longitudinally examine the physical activity levels and mental well-being of Scottish students during their first year at university. Methods: Participants were 48 first year students (males, $n=24$; females, $\mathrm{n}=24$ ) aged 18-19 years enrolled at two Scottish universities in the UK. At entry to university (semester 1) participants completed a validated self-report measure of physical activity and measures of anxiety and depression. Participants' physical activity levels were assessed for seven days via accelerometry. Participants completed the same measures six months later (semester 2). Results: Daily moderate physical activity levels decreased across the semesters yet perceptions of mental well-being remained stable. There was a significant increase in hip and waist circumferences across the semesters. Conclusions: First year of university is a critical period for establishing positive health behaviours and there is a need for universities to actively encourage physical activity as an integral part of university life.

23 Keywords: higher education, physical activity, university, students, mental well-being, health 
The physical and mental health benefits of engaging in regular physical activity are well established for adults (Reiner et al. 2013, Teychenne, Ball, and Salmon 2008). However physical activity levels in young adults decline between the ages of 18 and 24 (Leslie et al. 2001) which coincides with a period where many young adults are involved in higher education. In 2004, Haase et al. (2004) conducted a cross-sectional survey examining the leisure-time physical activity levels of 19,298 university students from 23 countries varying in culture and level of economic development. Comparative to data in the United States (US) and Australia, data examining physical activity levels of university students in the United Kingdom (UK) highlighted that $73 \%$ of males and $79 \%$ of females did not meet the physical activity guidelines at the time. Since then, UK physical activity guidelines for adults have been updated to a minimum of 150 minutes of moderate intensity physical activity over seven days (Department of Health 2011). Therefore, despite previous research indicating high levels of inactivity amongst the student population in several countries, these data are dated and comparative to previous guidelines; based on a cross-sectional survey accounting solely for leisure-time physical activity and are only representative of university students in England. Furthermore, studies that have compared the physical activity levels of students using subjective and objective measurement tools have shown that students may overestimate their physical activity levels (Bray and Born 2004, Loney et al. 2011, Downs et al. 2014) which suggests students perceive themselves to be engaging in desirable health behaviours whilst objective data suggests this may not be the case.

During the transition from school to higher education, young adults experience a substantial amount of change where they progress from the highly controlled setting of school to the autonomous and self-motivated environment of university (MacNamara and 
49 Collins 2010). This shift in independence allows students more choice and control over a range of health behaviours, including physical activity (Bray and Born 2004). Research has suggested that relocation to on-campus accommodation in first year at university could further contribute to a reduction in students' physical activity levels. A systematic review by Irwin (2004) compared the physical activity levels of 35,747 students living on-campus and off-campus across 27 countries. The authors concluded that overall those students who lived on campus were less active than those who lived off campus, suggesting that the campus environment is a key determinant of physical activity choices and behaviours. More recent findings (King et al. 2011) also showed a larger reduction $(M=-47 \%)$ in physical activity levels in those students who relocated to on-campus accommodation compared to those students who did not relocate $(\mathrm{M}=-33 \%)$. However all data available comparing the physical activity levels of students who reside on-campus and off campus are gathered using subjective measures and there are limited data for UK university students, particularly those that attend universities outside of England.

In order to address low levels of physical activity amongst students, several health promoting university initiatives have been implemented in recent years (e.g., Freshman 5 to Thrive: Creating Opportunities for Personal Empowerment and Healthy Lifestyles; Melnyk et al. 2014). McDaniel, Melton, and Langdon (2014) developed a Physical Activity Passport (PAP) as part of an intervention to increase students' physical activity levels through combining student life with academics. The PAP intervention was implemented as a university assignment where printed physical activity logs recorded physical activity levels that were signed off by fitness class instructors or sports captains, with the aim to increase physical activity levels outside academic study. Findings identified that those students who took part in the PAP intervention attended four times as many group fitness classes $(M=$ $6.98, S D=0.38$ number of fitness classes/week) than those students who were in the control 
group ( $M=1.48, S D=0.26$ number of fitness classes/week). Encouraging students to adopt an active lifestyle through such initiatives whilst at university could help them develop autonomy over health behaviours.

In addition to the potential relocation from home, the transition from school to university involves a number of changes that can impact on the mental well-being of students including financial concerns, academic pressures and a lack of social support. It could be argued that the financial concerns associated with tuition fees, living costs and associated costs of studying can be a significant source of stress for many students (Nelson et al. 2008, Lange and Byrd 1998). In 2010 the National Union of Students in Scotland released a report on Scottish students' mental well-being (Scotland 2010). The survey was completed by over 1800 students from 19 colleges and 15 universities across Scotland and having enough money for day to day living' was reported as a key source of stress for almost $70 \%$ of students. In the report, academic pressures such as exams and assessments were reported as the greatest source of stress for university students with over $90 \%$ of students stating academic pressures caused them more stress than expected.

Studying at university offers the opportunity to establish new social networks for many young adults yet the pressure to make friends and integrate into university life could be a source of worry and anxiety. Longitudinal survey data on the prevalence of mental health problems in a sample of 763 US college students was collected by Zivin et al. (2009). Findings indicated that over the two year period, over one third of the sample had a mental health problem and this persisted in $60 \%$ of the sample during their time at college. This is comparable to UK data where Berwick and colleagues (Bewick et al. 2010) reported temporal changes in psychological well-being of UK students at pre-registration and progression throughout their university degree. The highest levels of anxiety were reported in semester 
one of second year and anxiety levels did not reduce to levels measured initially at preregistration. Although consistently lower than the reported levels of anxiety across the three years, levels of depression steadily increased, with the highest levels of depression reported in semester two of third year with levels almost double that reported at pre-registration. Overall the results suggest that university is predominately an anxiety-provoking rather than a depressive time and levels of anxiety and depression at university are overall higher than prior to entering higher education.

The health of the student population is a public health concern as the transition from late adolescence to adulthood is accompanied by a time of increased personal choice, and behaviours adopted during this time may develop into long-term habits. This coincides with a period of change where financial concerns and academic and social pressures can cause psychological distress for students as they enter university. It is well established that engaging in regular physical activity can improve psychological well-being and should be considered as an important part of university life. The overall aim of the study was to examine the physical activity levels and mental well-being of Scottish students during their first year at university. A secondary aim was to examine the effect of gender and living environment (i.e. on-campus vs. off-campus) on physical activity levels and mental wellbeing.

\section{Methods}

\section{Participants}

Participants were 52 students (males, $n=26$; females, $\mathrm{n}=26)$ aged $18-19$ years $(M=$ $18.5 \pm 0.8$ ) in their first year of undergraduate degree programmes at two Scottish universities in the UK. Participants were recruited through poster advertisement at both universities, 
email distribution lists and through word of mouth. The study was granted institutional ethical approval and written informed consent was provided before data collection began with additional verbal information given to participants on the first visit to the laboratory. The first

124 phase of data collection took place during the first academic semester of first year (September 125 2013), referred to as 'Semester 1'. The second phase of data collection took place at the beginning of the second academic semester of first year (January 2014), referred to as 'Semester 2'.

\section{Procedures}

The study procedures involved two visits to the university laboratory in each semester. On the initial visit participants provided informed consent and were then asked to complete a demographic questionnaire and the Hospital Anxiety and Depression Scale (HADS)(Zigmund and Snaith 1983). Participants' height, mass, blood pressure, waist and hip circumference were then assessed. Once participants completed all questionnaires and physical assessments, they were fitted with a tri-axial accelerometer (GT3x Actigraph) and instructed on how to wear it. Participants were asked to wear the accelerometer for 7 days to assess the frequency, duration and intensity of their daily activity. After the 7 days, the participants visited the laboratory to return their accelerometer and non-wear time log diary. Participants also completed the International Physical Activity Questionnaire (IPAQ)(Craig et al. 2003) to assess their self-reported moderate and vigorous physical activity levels during the previous week.

\section{Measures}


Participants were asked to complete a demographic questionnaire to assess gender, age, ethnicity, undergraduate degree course and current living environment. Students who lived in university halls of residence were categorized as 'living on-campus' and those who remained living at home or lived in alternative accommodation were categorized as 'living off-campus'. A majority of the student sample lived in on-campus accommodation $(n=37$ : $71.2 \%)$ compared to the amount that lived in off-campus accommodation ( $n=15: 28.8 \%)$. For all the students included in the study neither the living environment nor the academic course being studied changed from semester 1 to semester 2 .

\section{Psychological well-being}

The HADS (Zigmund and Snaith 1983) is a self-report questionnaire used frequently to assess anxiety and depression, which requires participants to recall how they have felt in the previous week. The HADS comprises a 14-item scale with seven questions relating to anxiety and seven questions relating to depression. Answers consist on a four point Likert scale; $0=$ not at all, $1=$ not often, $2=$ quite a lot, $3=$ definitely. Scores are summed where a score of 0-7 signifies no presence of clinical symptoms; 8-10 indicates mild symptoms; 11-14 demonstrates moderate symptoms of depression and anxiety and a score of 15-21 indicates severe symptoms. Published UK normative data (Crawford et al. 2001) reported a HADS depression score as 3.68 and HADS anxiety score as 6.14 out of a maximum score of 21 . Previous research suggests that the HADS is a widely used and valid measure of depression and/or anxiety (Bjelland et al. 2002).

\section{Physical measurements}

Physical assessments included recording of participants' height, mass, blood pressure, hip and waist circumference. Height was recorded using a Stadiometer (Model 225, Seca Ltd) 
and mass was assessed using precision scales (Model 770, Seca Ltd). Participants' systolic

167 and diastolic blood pressures were recorded using an Omron 2 automatic blood pressure monitor, which was placed on the participant's relaxed left upper arm. Waist and hip circumferences were measured using a tape measure and participants were asked to position their feet together to ensure consistency in measurements. Waist and hip circumferences were measured in accordance with the International Society for the Advancement of Kinanthropometry standardized protocols.

\section{International Physical Activity Questionnaire}

Participants were asked to complete the IPAQ (Craig et al. 2003) which is used to assess the physical activity levels of participants over the previous 7 days. The IPAQ assesses physical activity undertaken across a comprehensive set of domains including leisure time, domestic and gardening activities, work-related and transport-related activity. The specific types of activity that are assessed are walking, moderate intensity activities and vigorous intensity activities. Frequency (measured in days per week) and duration (time per day) are collected separately for each specific type of activity. Previous research confirms the IPAQ to have good construct validity when used alongside objective physical activity monitors and self-report diaries (Hagstromer, Oja, and Sjostrom 2006).

\section{ActiGraph Accelerometer}

A 19g tri-axial accelerometer GT3X+ (ActiGraph, LLC, Pensacola, FL, USA) was used to assess the participants' objective physical activity levels through measuring frequency, intensity and duration of activity. The accelerometer was attached to an adjustable

187 elastic belt and worn on the participant's right hip for 7 consecutive days. Each participant was provided with thorough instruction on how to wear the ActiGraph and asked to fill in an 
activity log to provide detail on when the device was removed (non-wear time) on each of the 7 days. The Freedson Adult VM3 (Sasaki, John, and Freedson 2011) cut points were used to determine the intensity of the activity being undertaken, measured as counts per minute (CPM). Activity monitors were set to a sample rate of 10 -second epochs, in accordance with previous research (Trost, McIver, and Pate 2005) and non-wear time was counted as 60 consecutive minutes with zero counts and excluded from further analysis. Data were included if the participants had accumulated a minimum of 10 hours of valid activity recordings per day for at least 4 of the 7 days, which is in accordance with similar studies (Trost, McIver, and Pate 2005, Hagstromer et al. 2010).

\section{Data Analysis}

All data were screened for normal distribution and homogeneity of variance and significance was accepted at $p<0.05$. Participants' objective accelerometer data were screened for any outliers to exclude any days that did not meet the inclusion criteria, resulting in the exclusion of four participants from the data analysis. Therefore comparisons between semester 1 and semester 2 were made on a sample of 48 male and female first year students.

Paired sample T-tests were used to examine changes in subjectively- and objectivelymeasured physical activity, physical characteristics, anxiety and depression between semester 1 and semester 2. A two-factor repeated-measures ANOVA was conducted to examine the influence of gender and living environment on calculated delta values (semester 1-semester 2 differences) in physical activity, physical characteristics, anxiety and depression. Effect sizes were estimated using Cohen's $d$ (Cohen 1988) interpretation guidelines of $d>=0.2$ (small effect size), $d>=0.5$ (medium effect size) and $d>=0.8$ (large effect size).

\section{Results}




\section{Changes in physical activity}

213

214

215

216

217

218

219

Accelerometer data across both semesters is provided in Table 1. There was an overall significant decrease in time spent in moderate intensity physical activity (average daily minutes) from semester 1 to semester $2(t=4.701, \mathrm{df}=47, p<.001)$. The mean difference between semester 1 and semester 2 was 10.8 minutes and the $95 \%$ confidence interval for the estimated population mean was between 15.4 minutes and 6.2 minutes. The effect size was small $(d=0.1)$. There was an overall increase in light intensity physical activity (percentage of daily physical activity) from semester 1 to semester $2(t=3.195, \mathrm{df}=47, p<.05)$. The mean difference between semester 1 and semester 2 was $-0.9 \%$ and the $95 \%$ confidence interval for estimated population mean difference was between $-0.4 \%$ and $-1.5 \%$. The effect size was small $(d=0.3)$. There was an overall decrease in the percentage of time spent in moderate intensity physical activity (percentage of daily physical activity) from semester 1 to semester $2(t=3.949, \mathrm{df}=47, p<.001)$. The mean difference between semester 1 and semester 2 was $1.0 \%$ and the $95 \%$ confidence interval for the estimated population mean was between $1.5 \%$ and $0.5 \%$. The effect size was medium $(d=0.5)$.

\section{** Insert Table 1 here h* $^{*}$}

Table 2 shows the self-report physical activity data based on the IPAQ. There were no significant differences between semester 1 and semester 2 in any of the IPAQ activities (walking; moderate physical activity; vigorous physical activity and total physical activity) or in any of the domains assessed by the IPAQ (work; transport, domestic; leisure).

\footnotetext{
**Insert Table 2 here**
}

\section{Changes in physical characteristics}



from semester $1(M=74.3 \mathrm{~cm} ; S D=6.7)$ to semester $2(M=76.2 \mathrm{~cm} ; S D=6.4)$. The mean difference between semester 1 and semester 2 was $+1.9 \mathrm{~cm}$ and the $95 \%$ confidence interval

237 for the estimated population mean difference was between $+0.9 \mathrm{~cm}$ and $+2.9 \mathrm{~cm}$. The effect

238 size was small $(d=0.3)$. There was also a significant increase in hip circumference $(t=$ $2393.263, \mathrm{df}=47, p<.01)$ from semester $1(M=95.3 \mathrm{~cm} ; S D=7.1)$ to semester $2(M=97.0 \mathrm{~cm}$; $240 S D=7.0)$. The mean difference between semester 1 and semester 2 was $+1.7 \mathrm{~cm}$ and the $95 \%$ 241 confidence interval for the estimated population mean difference was between $+0.6 \mathrm{~cm}$ and $242+2.7 \mathrm{~cm}$. The effect size was small $(d=0.2)$. There were no significant differences between semester 1 and semester 2 for height, mass, systolic and diastolic blood pressure.

Changes in anxiety and depression semester $1(M=6.8 ; S D=3.9)$ to semester $2(M=4.4 ; S D=2.4)$. The mean difference between semester 1 and semester 2 was 2.4 and the $95 \%$ confidence interval for the estimated population mean difference was between 3.3 and 1.5. The effect size was large $(d=0.8)$. There was no significant difference in levels of depression from semester 1 to semester 2 . environment on the delta values of the outcome variables.

\section{Discussion}



were exceeding the current UK physical activity guidelines (Department of Health 2011) accumulating nearly 60 minutes of daily moderate to vigorous physical activity (MVPA). This is an encouraging finding yet needs to be interpreted with caution. Previous research has identified the potential of reactivity which can often lead to temporary increases in physical activity among individuals wearing an accelerometer for a period of 7 days (Motl, McAuley, and Dlugonski 2012). The finding contradicts recent accelerometer data collected in a sample of US college students (Downs et al. 2014) where only $33 \%$ of the sample $(n=72)$ were meeting US physical activity guidelines, accumulating 20 minutes of daily MVPA. However it is important to note that the data were collected over a period of 14 days (Downs et al. 2014) which may have reduced the likelihood of reactivity compared to the 7 day measurement period in our study.

Over the six month period, there was a significant decrease in the amount of time spent in moderate physical activity and a significant increase in time spent in light physical activity. This suggests that students may be spending more time in light intensity physical activity to the detriment of engaging in moderate physical activity. Whilst this was only examined over two time points in their first year at university, a continuous trend in decreasing daily moderate intensity physical activity could reduce the potential of the associated health benefits of moderate physical activity during their time spent at university.

274 There is limited comparative objective longitudinal data on student physical activity levels yet there is partial evidence of a shift in the type and intensity of activity students participate in whilst at university (Racette et al. 2005). data. Although not statistically significant, students' perceptions of time spent in moderate 
and vigorous physical activity decreased across the two semesters and overall daily physical activity decreased. This suggests that students are meeting the current UK physical activity guidelines yet are overestimating the amount of time spent in daily vigorous physical activity ( $M=42.1 \mathrm{mins})$ when compared to the objective data of the accelerometer $(M=8.1 \mathrm{mins})$. Overestimation of duration and intensity of physical activity is commonplace when relying on self-report measures (Montoye et al. 1996). Similar findings were reported by Downs and colleagues (Downs et al. 2014) in their sample of 72 US students who reported spending 68 minutes per day in MVPA yet accelerometer data estimated they spent 20 minutes per day in MVPA. Overall the findings point towards a need for surveillance data in this population to consider the inclusion of objective assessment methods and for health promotion initiatives within universities to increase knowledge and awareness of the intensities of activities that are offered to students.

Weight gain amongst students, particularly during the freshman year, has been studied extensively in the US and Canada (Holm-Denoma et al. 2008, Pullman et al. 2009, Racette et al. 2008) reporting increases in body mass and body mass index in this population. In this study, there were significant increases in waist and hip circumference over the six months. Our findings share similarities with previous literature looking at changes in body composition during the initial stages of university study. Body composition was assessed in 120 students in their freshman year and then in their sophomore year (Gropper et al. 2011) and results indicated a significant increase in waist circumference $(M=+0.9 \mathrm{~cm})$, comparable to the increases reported in our study $(M=+1.9 \mathrm{~cm})$. Increases in weight gain and waist and hip circumference could be attributed to the reduced time spent in moderate physical activity or changes in dietary habits, however dietary habits were not monitored in the current study. These significant increases in anthropometric measurements in a six month period is concerning and need to be prioritised in health promotion initiatives targeting students. 
For young adults, the transition to university is a period characterised by change, uncertainty and adjustment in several aspects of their lives (e.g., financial, social, academic) with the potential to impact upon their psychological well-being (Bray and Born 2004). In the current study, levels of anxiety significantly decreased from semester 1 to semester 2 , perhaps suggesting that initial entry to university is an anxiety provoking time yet anxiety lessens as students adjust to their new environment. Similar findings have been documented in a study examining the temporal nature of psychological well-being of 4,699 students entering their first year at university in the UK (Cooke et al. 2006). Data were collected over four time-points (pre-entry to university; one-month after entry; end of semester 1; end of semester 2) and indicated that levels of anxiety were heightened upon initial entry to university when compared to both pre-entry to university levels and levels of anxiety at the end of semester 2. In the current study, reported levels of depression did not significantly increase across the two semesters and depression scores were comparatively low in relation to levels of anxiety. This was also evident in the data collected by Cooke and colleagues who suggested that the first year of university is a time of heightened anxiety yet not a particularly depressive time for students. Overall the findings reinforce the need for universities to monitor changes in the psychological well-being of students during their time at university and to provide adequate support for first year students as this appears to be a period of critical adjustment where they experience increased levels of anxiety.

Previous research has suggested that a change in the physical environment could impact on the physical activity levels of students (Irwin 2004, Kapinos and Yakusheva 2011, King et al. 2011) and that students living on campus were less active than those living in offcampus accommodation (Irwin 2004). Yet recently, a study examining how living on or off campus is associated with dietary and physical activity patterns in college students across seven semesters found that living off campus exacerbated decreases in physical activity and 
poor dietary consumption (Small et al. 2013). Contrary to the above studies, we found that the decrease in physical activity levels, increase in waist and hip circumference and decrease in anxiety levels were not influenced by living environment. It is important to note that the majority of the sample lived on-campus which may have limited the possible influence of living environment and needs further consideration. Contrary to a recent longitudinal study (Deforche et al. 2015) examining changes in body mass, physical activity, sedentary behaviour and dietary intake during the transition to higher education in 291 students, we also found no main effect of gender in any of the changes in the outcome measures across the two semesters yet physical activity was not assessed using an objective method, which makes it difficult to draw comparisons.

Whilst the study had identifiable strengths in the longitudinal assessment of the physical activity levels in first year students based at two universities using objective and subjective measures, there were also limitations. Our sample represented a very small proportion of the first year students at the universities who self-selected for the study and only included full-time undergraduate students, which limit any generalisations that can be made for part-time students. Gaining a detailed understanding of the entry to university as a transition experience requires us to examine behaviours prior to starting university. Similarly, longitudinal assessment of physical activity, anxiety and depression beyond first year at university would provide a clearer representation of temporal fluctuations in these health outcomes across time spent at university. Finally, it would be useful to compare the health behaviours of students with those of young adults who enter employment, as opposed to attending university, to determine the impact of university life on student health and wellbeing.

\section{Conclusions}


354 yet the transition to university is a period characterised by change, uncertainty and adjustment

355 that can have a negative impact on health and well-being. Our findings indicated that daily

356 moderate physical activity levels decreased across the semesters in first year and that students

357 spent more time engaging in light intensity activities in semester 2 . There were also increases 358 in waist and hip circumference during their first year at university yet these changes were not 359 influenced by gender or living environment. Future research could explore other potential 360 predictors of the change in physical activity levels during first year. Unfavourable changes in 361 physical activity levels and physical characteristics in our first year student sample over a 362 relatively short period of time (six months) are therefore concerning. There is a need for universities to actively encourage physical activity as an integral part of university life, providing opportunities for physical activity and to enhance students' knowledge on the importance of MVPA. Entry to university appeared to be a time of heightened anxiety for our student sample yet levels of depression reported were comparably low. Signposting students

367 to the relevant support services upon entry to university is recommended to assist students 368 during this transitional period. It was also apparent that psychological well-being is changeable even over a short period of time and needs to be monitored throughout time spent at university, to allow the provision of appropriate and timely intervention. 
Table 1. Daily accelerometer physical activity data across semesters 1 and 2

\begin{tabular}{|c|c|c|c|c|}
\hline & \multicolumn{2}{|c|}{ Semester $1(n=48)$} & \multicolumn{2}{|c|}{ Semester $2(n=48)$} \\
\hline & $M$ & $S D$ & $M$ & $S D$ \\
\hline Total time in activity (mins) & 867.9 & 59.0 & 845.4 & 64.8 \\
\hline Light activity (mins) & 794.2 & 52.3 & 782.3 & 66.5 \\
\hline Moderate activity (mins) & 64.8 & 21.9 & $54.0 * *$ & 17.6 \\
\hline Vigorous activity (mins) & 8.0 & 8.8 & 8.2 & 7.6 \\
\hline Very vigorous activity (mins) & 1.1 & 1.3 & 1.0 & 1.5 \\
\hline$\%$ time in light activity & 91.6 & 2.8 & $92.5^{*}$ & 2.5 \\
\hline$\%$ time in moderate activity & 7.4 & 2.3 & $6.4 * *$ & 2.1 \\
\hline$\%$ time in vigorous activity & 0.9 & 1.0 & 0.9 & 0.8 \\
\hline $\begin{array}{l}\% \text { time in very vigorous } \\
\text { activity }\end{array}$ & 0.1 & 0.1 & 0.2 & 0.1 \\
\hline \multicolumn{5}{|c|}{$\begin{array}{l}*_{p}<.05 ; \text { significant difference between semesters } \\
*_{\mathrm{p}}<.001 ; \text { significant difference between semesters }\end{array}$} \\
\hline & \multicolumn{2}{|c|}{ Semester $1(n=48)$} & \multicolumn{2}{|c|}{ Semester $2(n=48)$} \\
\hline & $M$ & $S D$ & $M$ & $S D$ \\
\hline Total activity (mins) & 156.3 & 104.4 & 144.9 & 91.0 \\
\hline Walking (mins) & 70.4 & 44.6 & 72.2 & 44.9 \\
\hline Moderate (mins) & 40.3 & 54.1 & 34.2 & 38.3 \\
\hline Vigorous (mins) & 45.7 & 39.2 & 38.5 & 28.7 \\
\hline Work-related activity (mins) & 22.2 & 55.1 & 19.4 & 43.6 \\
\hline Transport-related activity (mins) & 51.1 & 31.2 & 49.5 & 34.4 \\
\hline Domestic-related activity (mins) & 15.1 & 19.3 & 15.6 & 22.2 \\
\hline Leisure-related activity (mins) & 68.0 & 47.0 & 60.4 & 36.8 \\
\hline
\end{tabular}


Bewick, B., G. Koutsopoulou, J. Miles, E. Slaa, and M. Barkham. 2010. "Changes in undergraduate students' psychological well-being as they progress through university." Studies in Higher Education 35 (6):633-645.

Bjelland, I., A. A. Dahl, T. T. Haug, and D. Neckelmann. 2002. "The validity of the Hospital Anxiety and Depression Scale. An updated literature review." J Psychosom Res 52 (2):69-77.

Bray, S. R., and H. Born. 2004. "Transition to University and Vigorous Physical Activity: Implications for Health and Psychological Well-Being." Journal of American College Health 52:181-188.

Cohen, J. 1988. Statistical power analysis for the behavioral sciences 2nd ed. Hillsdale, NJ: Erlbaum.

Cooke, R., B. Bewick, M. Barkham, M. Bradley, and K. Audin. 2006. "Measuring, monitoring and managing the psychological well-being of first year University students." British Journal of Guidance and Counselling 34:505-517.

Craig, C. L., A. L. Marshall, M. Sjostrom, A. E. Bauman, M. L. Booth, B. E. Ainsworth, M. Pratt, U. Ekelund, A. Yngve, J. F. Sallis, and P. Oja. 2003. "International physical activity questionnaire: 12-country reliability and validity." Med Sci Sports Exerc 35 (8):1381-95. doi: 10.1249/01.MSS.0000078924.61453.FB.

Crawford, J. R., J. D. Henry, C. Crombie, and E. P. Taylor. 2001. "Normative data for the HADS from a large non-clinical sample." Br J Clin Psychol 40 (Pt 4):429-34.

Deforche, B., D. Van Dyck, T. Deliens, and I. De Bourdeaudhuij. 2015. "Changes in weight, physical activity, sedentary behaviour and dietary intake during the transition to higher education: a prospective study." Int J Behav Nutr Phys Act 12 (1):16. doi: 10.1186/s12966-015-0173-9.

Department of Health. 2011. Start Active, Stay Active. A report on physical activity for health from the four home countries' Chief Medical Oficers. edited by Department of Health.

Downs, A., J. V. Hoomissen, A. Lafrenz, and D. Julka. 2014. "Accelerometer-measured versus self-reported physical activity in college students: Implications for research and practice. ." Journal of American College Health 62 (3):201-212.

Gropper, S. S., A. Newton, P. Harrington, K. P. Simmons, L. J. Connell, and P. Ulrich. 2011. "Body composition changes during the first two years of university." Prev Med 52 (1):20-2. doi: 10.1016/j.ypmed.2010.10.008.

Haase, A., A. Steptoe, J. F. Sallis, and J. Wardle. 2004. "Leisure-time physical activity in university students from 23 countries: associations with health beliefs, risk awareness, and national economic development." Prev Med 39 (1):182-90. doi: 10.1016/j.ypmed.2004.01.028.

Hagstromer, M., P. Oja, and M. Sjostrom. 2006. "The International Physical Activity Questionnaire (IPAQ): a study of concurrent and construct validity." Public Health Nutr 9 (6):755-62.

Hagstromer, M., R. P. Troiano, M. Sjostrom, and D. Berrigan. 2010. "Levels and patterns of objectively assessed physical activity--a comparison between Sweden and the United States." Am J Epidemiol 171 (10):1055-64. doi: 10.1093/aje/kwq069.

Holm-Denoma, J. M., T. E. Joiner, K. D. Vohs, and T. F. Heatherton. 2008. "The "freshman fifteen" (the "freshman five" actually): predictors and possible explanations." Health Psychol 27 (1 Suppl):S3-9. doi: 10.1037/0278-6133.27.1.S3.

Irwin, J. D. 2004. "Prevalence of university students' sufficient physical activity: a systematic review." Percept Mot Skills 98 (3 Pt 1):927-43. doi: 10.2466/pms.98.3.927-943. 
Kapinos, K. A., and O. Yakusheva. 2011. "Environmental influences on young adult weight gain: evidence from a natural experiment." J Adolesc Health 48 (1):52-8. doi: 10.1016/j.jadohealth.2010.05.021.

King, S., R. Garrett, A. Wrench, and N. Lewis. 2011. "The loneliness of relocating: Does the transition to University pose a significant health risk for rural and isolated students?" First Year in Higher Education.

Lange, Clare, and Mark Byrd. 1998. "The Relationship Between Perceptions of Financial Distress and Feelings of Psychological Well-being in New Zealand University Students." International Journal of Adolescence and Youth 7 (3):193-209. doi: 10.1080/02673843.1998.9747824.

Leslie, E., M. J. Fotheringham, N. Owen, and A. Bauman. 2001. "Age-related differences in physical activity levels of young adults." Med Sci Sports Exerc 33 (2):255-8.

Loney, T., M. Standage, D. Thompson, S. J. Sebire, and S. Cumming. 2011. "Self-report vs. objectively assessed physical activity: which is right for public health?" J Phys Act Health 8 (1):62-70.

MacNamara, A., and D. Collins. 2010. "The role of psychological characteristics in managing the transition to university." Psychology of Sport and Exercise 11:353-362.

McDaniel, Tyler, Bridget F Melton, and Jody Langdon. 2014. "Promoting physical activity through student life and academics." Health Education Journal 73 (2):237-244. doi: 10.1177/0017896912471037.

Melnyk, Bernadette, Stephanie Kelly, Diana Jacobson, Kimberly Arcoleo, and Gabriel Shaibi. 2014. "Improving physical activity, mental health outcomes, and academic retention in college students with Freshman 5 to thrive: COPE/Healthy lifestyles." Journal of the American Association of Nurse Practitioners 26 (6):314-322. doi: 10.1002/2327-6924.12037.

Montoye, H. J., H. Kemper, W. Saris, and R. Washburn. 1996. Measuring physical activity and energy expenditure. Champaign, IL: Human Kinetics.

Motl, R. W., E. McAuley, and D. Dlugonski. 2012. "Reactivity in baseline accelerometer data from a physical activity behavioral intervention." Health Psychology 31 (2):172175.

Nelson, Melissa C., Katherine Lust, Mary Story, and Ed Ehlinger. 2008. "Credit Card Debt, Stress and Key Health Risk Behaviors Among College Students." American Journal of Health Promotion 22 (6):400-407. doi: doi:10.4278/ajhp.22.6.400.

Pullman, A. W., R. C. Masters, L. C. Zalot, L. E. Carde, M. M. Saraiva, Y. Y. Dam, J. A. Randall Simpson, and A. M. Duncan. 2009. "Effect of the transition from high school to university on anthropometric and lifestyle variables in males." Appl Physiol Nutr Metab 34 (2):162-71. doi: 10.1139/H09-007.

Racette, S. B., S. S. Deusinger, M. J. Strube, G. R. Highstein, and R. H. Deusinger. 2008. "Changes in weight and health behaviors from freshman through senior year of college." J Nutr Educ Behav 40 (1):39-42. doi: 10.1016/j.jneb.2007.01.001.

Racette, S., S. Deusinger, M. Strube, G. Highstein, and R. Deusinger. 2005. "Weight changes, exercise, and dietary pat- terns during freshmen and sophomore years of college." Journal of American College Health 53:245-251.

Reiner, M., C. Niermann, D. Jekauc, and A. Woll. 2013. "Long-term health benefits of physical activity--a systematic review of longitudinal studies." BMC Public Health 13:813. doi: 10.1186/1471-2458-13-813.

Sasaki, J. E., D. John, and P. S. Freedson. 2011. "Validation and comparison of ActiGraph activity monitors." J Sci Med Sport 14 (5):411-6. doi: 10.1016/j.jsams.2011.04.003.

Scotland, National Union of Students. 2010. Silently stressed: A survey into student mental wellbeing. Edinburgh: Healthier Scotland. 
Small, M., L. Bailey-Davis, N. Morgan, and J. Maggs. 2013. "Changes in eating and physical activity behaviors across seven semesters of college: living on or off campus matters." Health Educ Behav 40 (4):435-41. doi: 10.1177/1090198112467801.

Teychenne, M., K. Ball, and J. Salmon. 2008. "Physical activity and likelihood of depression in adults: a review." Prev Med 46 (5):397-411. doi: 10.1016/j.ypmed.2008.01.009.

Trost, S. G., K. L. McIver, and R. R. Pate. 2005. "Conducting accelerometer-based activity assessments in field-based research." Med Sci Sports Exerc 37 (11 Suppl):S531-43.

Zigmund, A., and R. Snaith. 1983. "The Hospitality Anxiety and Depression Scale." Acta Psychiatrica Scandinavia 67:361-370.

Zivin, Kara, Daniel Eisenberg, Sarah E. Gollust, and Ezra Golberstein. 2009. "Persistence of mental health problems and needs in a college student population." Journal of Affective Disorders 117 (3):180-185. doi: http://dx.doi.org/10.1016/j.jad.2009.01.001.

489 